\title{
ATOMIC DATA AND CORONAL DIAGNOSTICS
}

\author{
HELEN E. MASON \\ Department of Applied Mathematics and Theoretical Physics \\ Silver Street, CAMBRIDGE CB3 9EW, UK
}

\begin{abstract}
.
Spectroscopic diagnostics play an important role in determining the electron density and temperature structure, element abundances and dynamic nature of different features in the solar atmosphere. To ensure that these techniques are accurate it is necessary to interface solar analysis programs with the best available atomic data calculations. Recent progress in this work is presented and applications to planned space observations are discussed. In particular, the CDS and SUMER instruments, on the Solar Heliospheric Observatory, will make extensive use of spectroscopic diagnostics to study the heating processes for the solar corona and the acceleration mechanisms for the solar wind.
\end{abstract}

\section{Introduction}

The Solar Heliospheric Observatory (SOHO) will be launched in 1995. It will carry several spectroscopic instruments including the Coronal Diagnostic Spectrometer (CDS, 160-800 $\AA$ ), the Solar Ultraviolet Measurement of

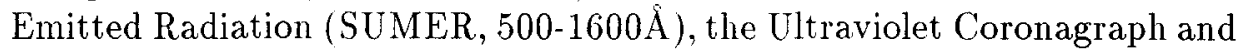
Spectrometer (UVCS), the Extreme Ultraviolet Imaging Telescope (EIT) and the Large Angle Spectroscopic Coronagraph (LASCO). Observations of the solar atmosphere will be carried out with high spatial, spectral and temporal resolution.

An UV spectroscopic diagnostics working group (co-ordinator, H.E. Mason) is preparing software and analysis techniques for CDS and SUMER. The topics covered include electron density and temperature determination, differential emission measures, elemental abundance variations and basic atomic data, both electron excitation, ionisation and recombination. 
Details of the instruments, science objectives and observing sequences can be found in the CDS blue book (editor R.A. Harrison) and the SUMER red book (editor K. Wilhelm). A review of VUV (100-2000 $)$ spectroscopic diagnostics for solar and stellar plasmas has recently been published in the Astronomy and Astrophysics Review by H.E. Mason and B. Monsignori Fossi (1994).

\section{Atomic Data Requirements}

The electron excitation rates required for SOHO spectra were the subject of an Atomic Data Assessment Workshop held in March 1992 (Atomic Data and Nuclear Data Tables, Vol. 57, editor J. Lang).

The Iron Project, an international collaboration of atomic physicists, aims to calculate new electron excitation rates using the close coupling approximation, with very accurate targets. The first stage is to compute collisional excitation rates for the fine structure transitions in the ground configuration of many ions. The second stage is to calculate new electron excitation rates for all the iron ions. A series of papers is being published in Astronomy and Astrophysics.

The Atomic Data and Analysis Structure (ADAS) package has been developed for fusion research by Summers at JET (Joint European Torus). ADAS provides an accurate method of atomic data analysis, storage and processing. It is being adapted for the analysis of CDS observations. ADAS solves the generalised collisional-radiative model for equilibrium or nonequilibrium conditions.

\section{Solar Analysis Programs}

A synthetic spectra in the UV wavelength for different solar models (quiet Sun, active region, coronal hole etc) is being developed by Dere, Monsignori Fossi, Mason and co-workers. The atomic rates are fitted using the Burgess and Tully (1992, Astron. Astrophys., 254, 436) assessment method.

Harrison and Thompson have reported (RAL-91-092) on the performance of a number of integral inversion codes used in the calculation of differential emission measure (DEM) from line intensities. These codes will be incorporated into the CDS software.

Electron density and temperature diagnostic line ratios will form an important part of the CDS and SUMER observations. It is necessary to specify sets of optimum spectral lines. The SERTS (Solar EUV Rocket Telescope and Spectrograph) spectra overlap in wavelength region with CDS and provide an excellent dataset for studying spectroscopic diagnostics.

HEM acknowledges the financial support of PPARC 